\title{
Enhancement of the ATLAS Trigger System with a Hardware Tracker Finder FTK
}

\author{
Jinlong Zhang

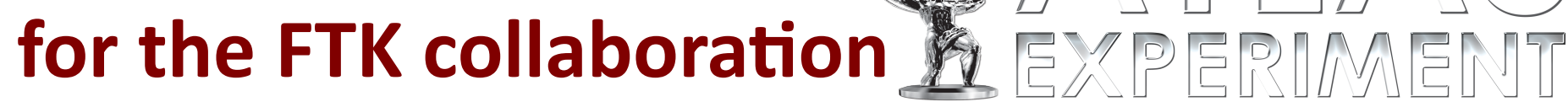




\section{Outline}

- Motivation

- FTK approach

- System overview and component functionality

- Performance

- Single particle efficiency and timing

- Identifying physics objects at $3 \times 10^{34}$

- Summary 


\section{Trigger with Tracks}

- Enhancement of the capability to examine the event characteristic at the LVL1 rate with all tracks

- Identification of heavy fermion objects originated from possible new physics scenarios (e.g., Higgs decay) in the enormous QCD jet background

- b-jets: displaced vertices or tracks with large impact parameter

- $\tau$-jets: 1 or 3 tracks in a narrow cone with a surrounding isolation region

- Effective lepton selection with tracking isolation 


\section{The challenge \& the Solution}

- The increasing LHC luminosity leads higher rate and larger event size.

- The trigger problem at high $\mathrm{P}_{\mathrm{T}}$ can't be solved by just increasing thresholds

- Suppression of the higher background rates will require more sophisticated algorithms in earlier trigger levels

- Pileup increases the need for tracking and its execution time

- With introducing FTK, the global tracking would be completed at the beginning of the Level-2 trigger (LVL2). Thus the LVL2 processing power can be used more on needed sophisticated algorithms. 


\section{ATLAS TDAQ+FTK}

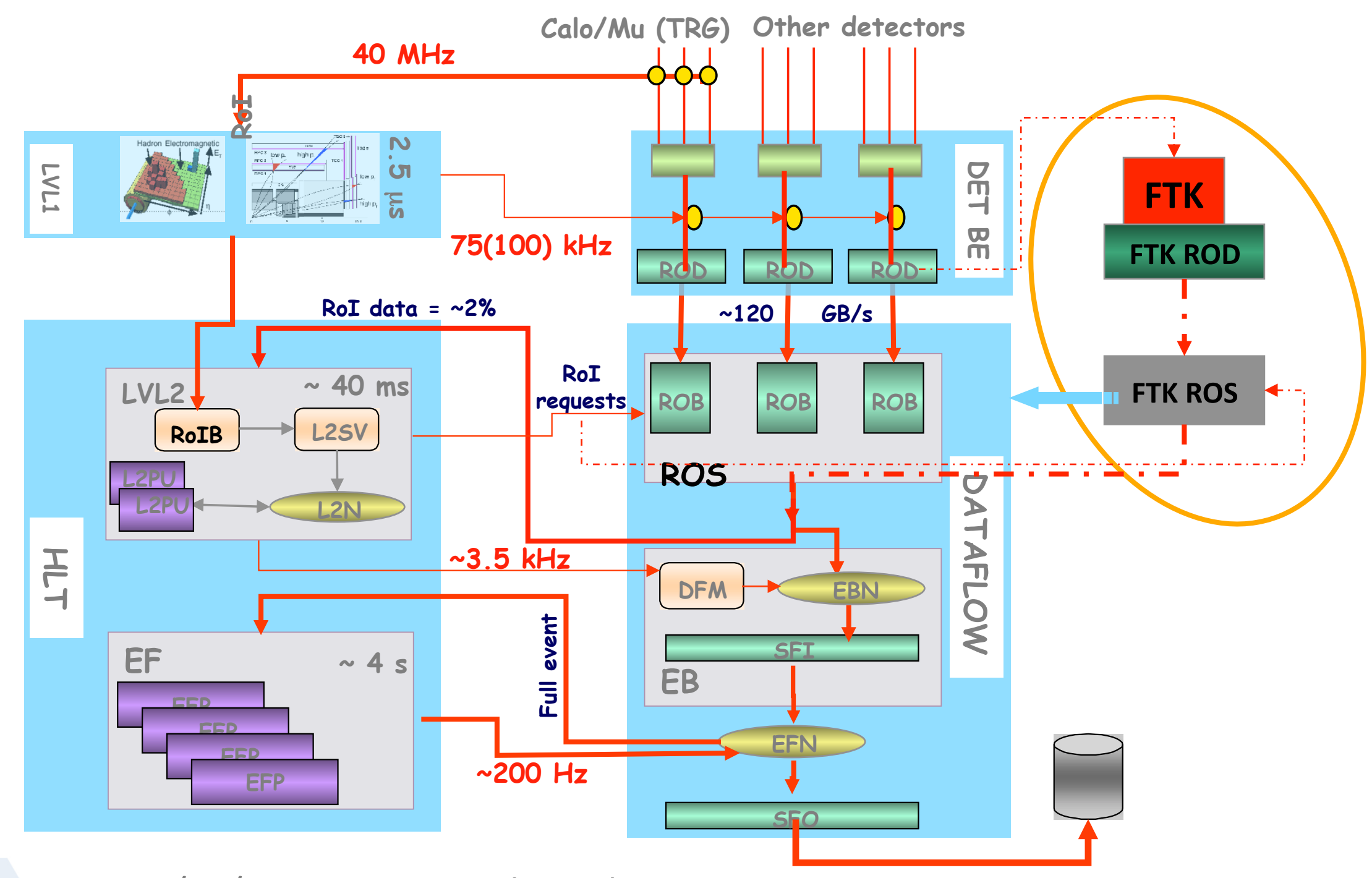

A TWEPP, 09/23/2010

Jinlong Zhang 


\section{Fast TracKer (FTK) Approach}

Use hardware to perform the global tracking in two steps pattern recognition and track fit
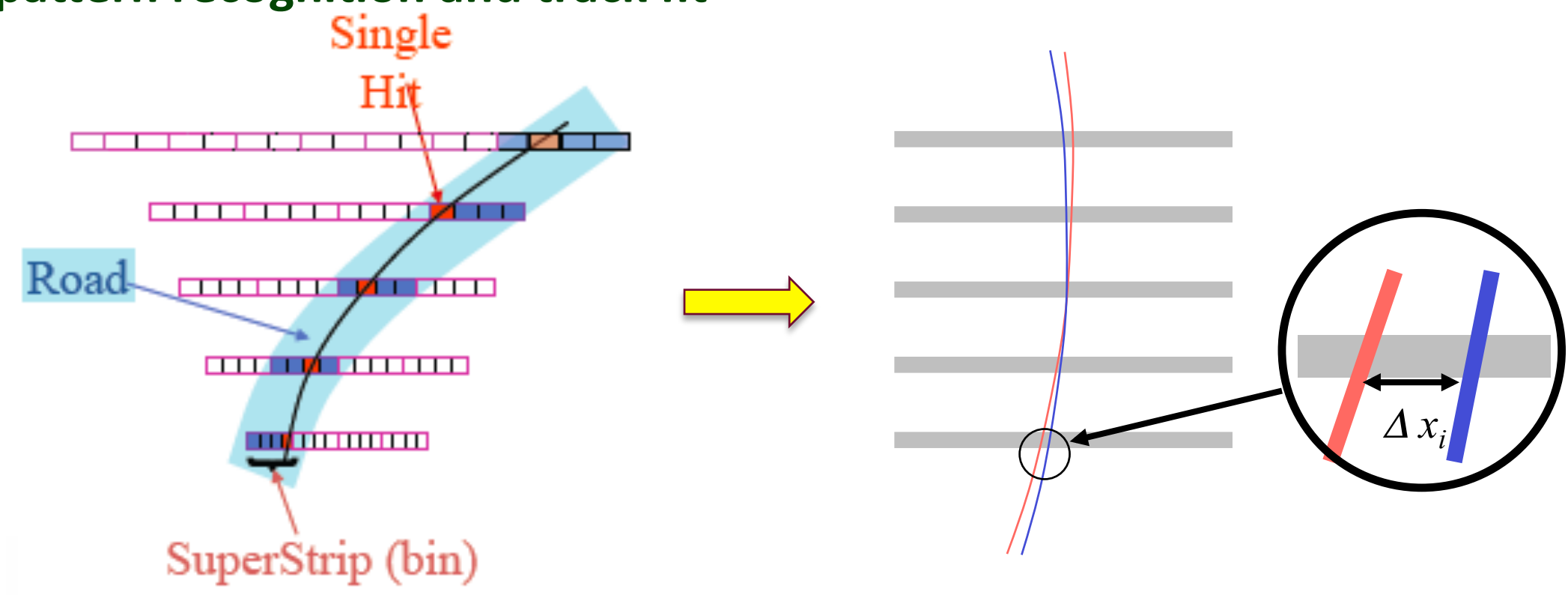

Pattern recognition in coarse resolution Track fit in full resolution (hits in a road) (superstrip $\rightarrow$ road) $F\left(x_{1}, x_{2}, x_{3}, \ldots\right) \sim a_{0}+a_{1} \Delta x_{1}+a_{2} \Delta x_{2}+a_{3} \Delta x_{3}+\ldots=0$

Road size to balance the workload between two steps 


\section{Pattern Recognition}

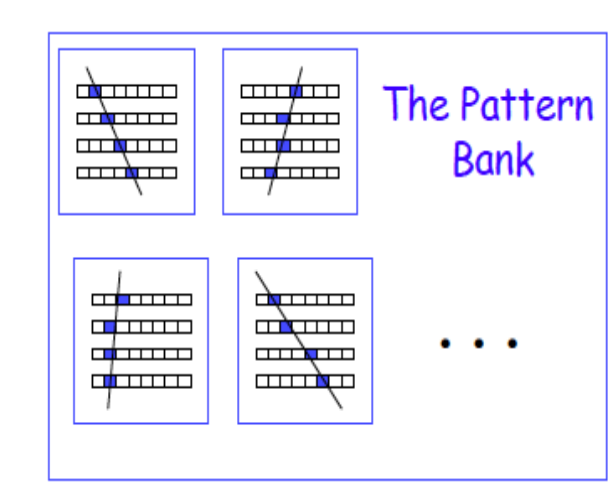

Prestored patterns $\left(10^{9}\right)$

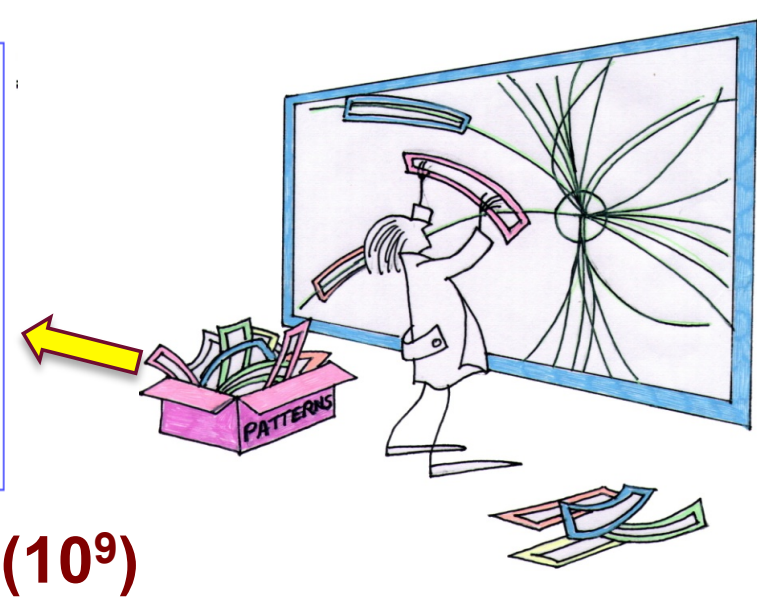

Content-addressable Memory (CAM)

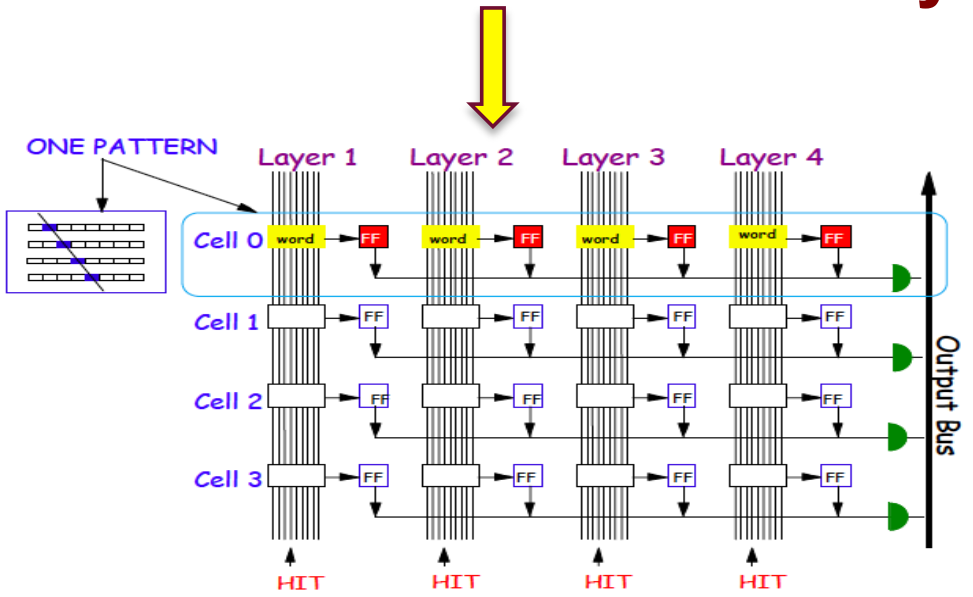

Fast pattern recognition
The Event

Hits of the event

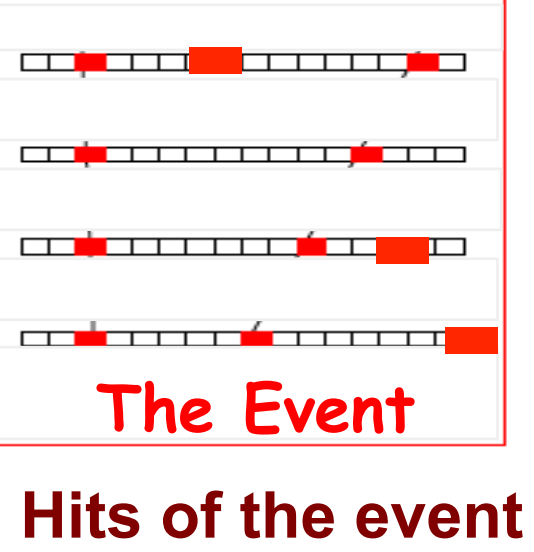

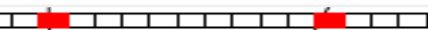

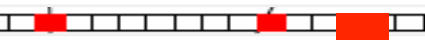

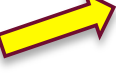

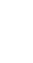




\section{Content-addressable Memory (CAM)}

- Take user data as input rather than the address

- Search the entire memory in a single operation

- Used often in network search elements

- Available commercially and in HEP custom design (limited)

- INFN AM able to identify correlation among input data words received on different clock cycles

\begin{tabular}{|c|c|c|}
\hline & AM INFN (2004) & CAM (latest) \\
\hline Availability & Non-commercial & Good \\
\hline Technology & $180 \mathrm{~nm}$ & $55 \mathrm{~nm}$ \\
\hline Speed & $40 \mathrm{MHz}$ & $500 \mathrm{MHz}$ \\
\hline Size & $(6 \times 16) \times 5 K$ & $36 \mathrm{X} 1024 \mathrm{~K}, 576 \mathrm{X} 64 \mathrm{~K}$ \\
\hline Max Channels & $384 K$ & 576 \\
\hline Flexibility & Low & High \\
\hline \multirow[t]{2}{*}{ Price } & $10-15 €$ & $O(100-200 €)$ \\
\hline & NNFN & NETL J GIC $_{\text {ilcostrtens }}$ \\
\hline
\end{tabular}

1) IEEE Trans.Nucl.Sci.53:2428-2433,2006

2) http://www.netlogicmicro.com/Products/Layer2/Layer2-3.htm 


\section{Track Fitting}

- Determine the helix parameters and $\chi^{2}$

- Fit with the local silicon hit coordinates (one module in each layer) in linear

$$
p_{i}=\sum_{j=1}^{14} a_{i j} x_{j}+b_{i}
$$

- $P_{i}:$ the helix parameters and $\chi^{2}$ components

$-X_{j}$ : the hit coordinates in the silicon layers

- $a_{i j} \& b_{i}$ : prestored constants determined from full simulation or real data track

- Very fast in DSPs ( $\sim 1$ ns per track) 


\section{System Overview}

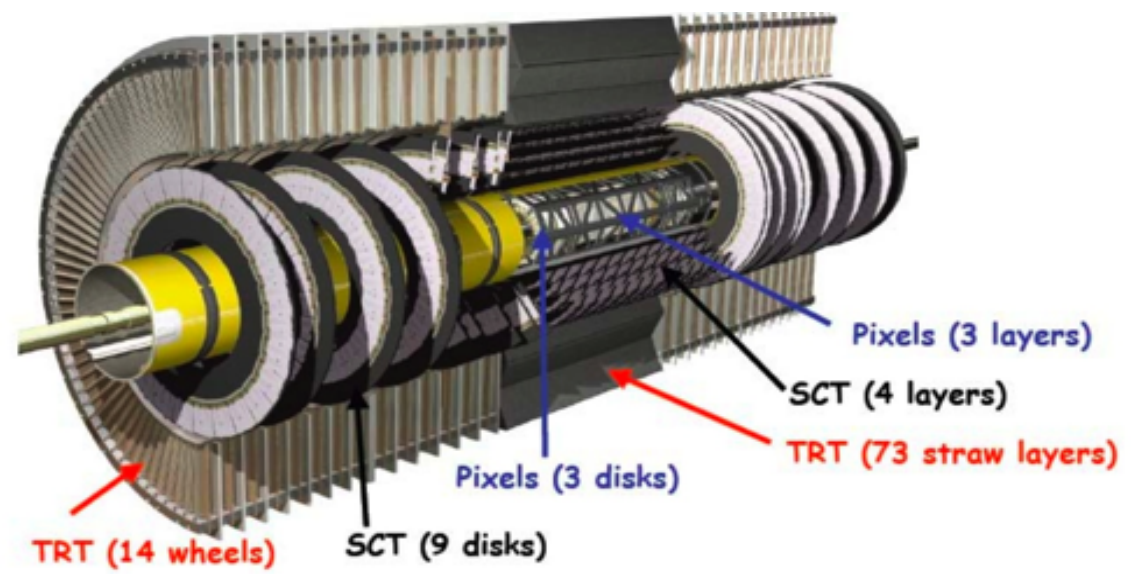

- PIX (3 layers ) \& SCT (4 double layers)

- Architecture of 11 layers in one step (PIX 3 + SCT 4 axial + SCT 4 stereo) unaffordable

- Options:

-7 layers (PIX $3+$ SCT 4 axial)

$-\mathrm{SCT}^{1 \text { st }}-\mathrm{PIX}^{2 \text { nd }}(8 \rightarrow 4)$

- 8 sectors, each with one crate, 8 (12) crates @ $10^{34}\left(3 \times 10^{34}\right)$ total - 4X2 $\eta-\phi$ towers in each sector, each tower with one slot unit

- Overlaps to maintain high efficiency

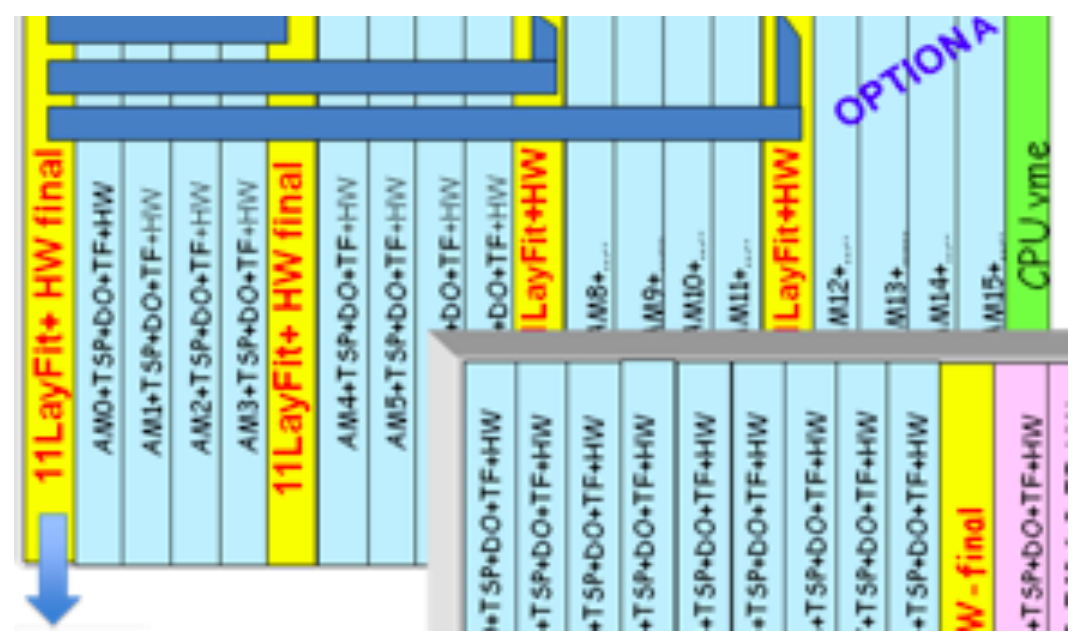




\section{System Functional Sketch}

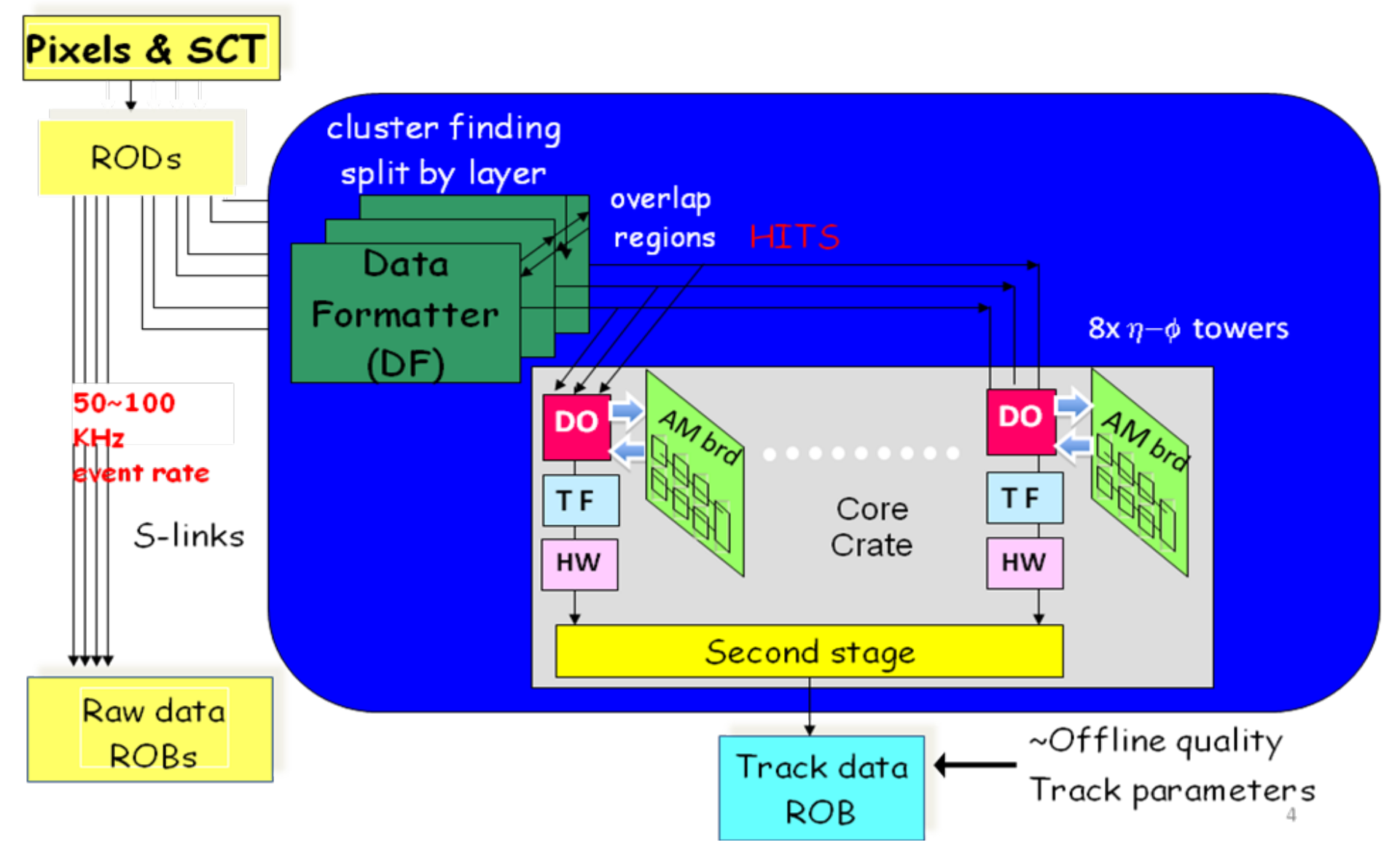




\section{Data Formatter (DF)}

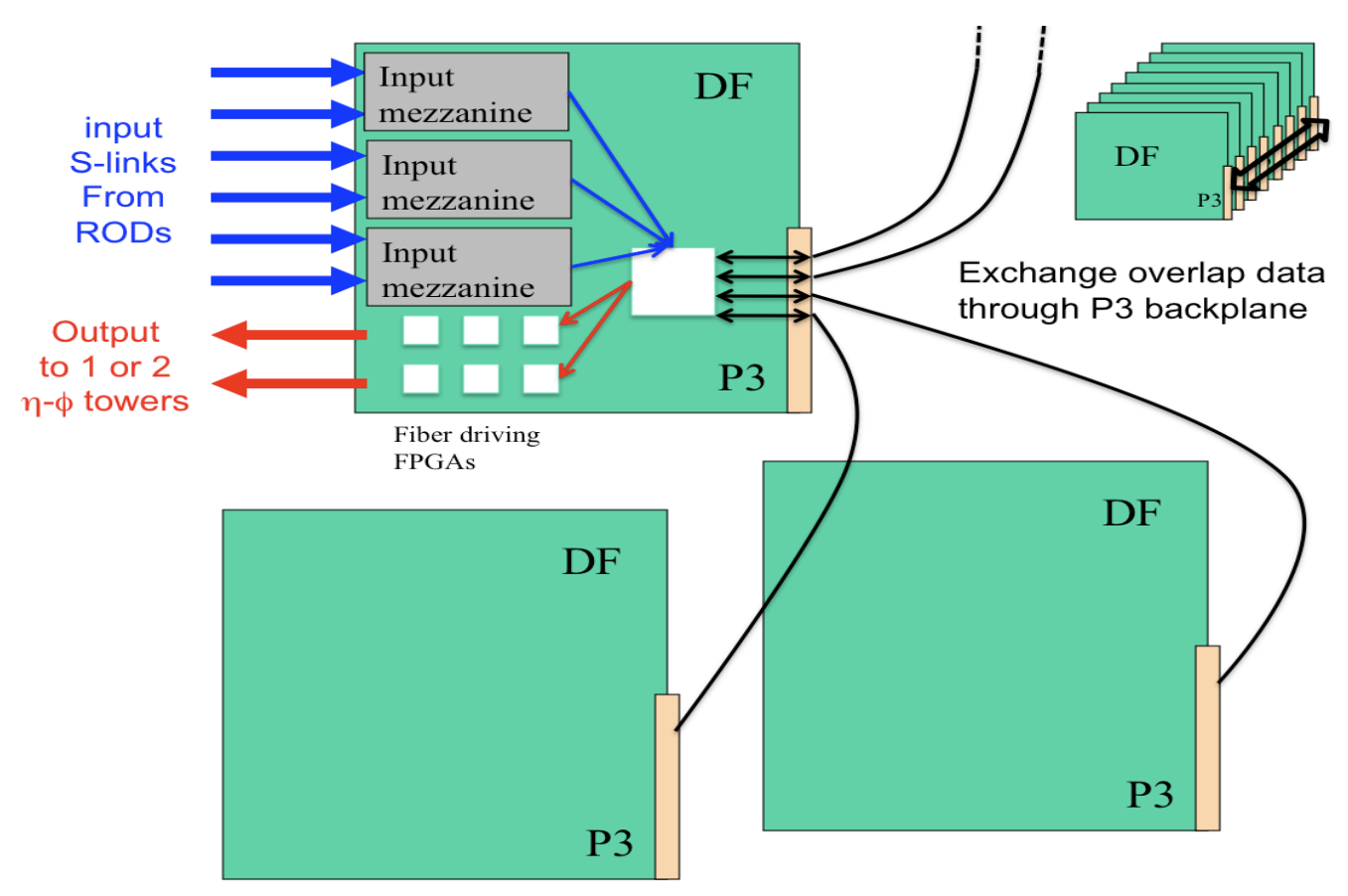

- Receive the silicon hits from the pixels and SCT

- Perform cluster finding (2D in pixels)

- Sends the cluster centroids to the appropriate $\eta-\phi$ towers in the core crates 


\section{Process Unit (AM board)}

- Contain 4 mezzanine cards, each connected to a separate DO, TF, HW chain.

- The mezzanine card holds 32 custom standard-cell content-addressable memory chips

- 3.7K patterns per existing AM chip (for 8 layers); $1.8 \mathrm{~W}$ power consumption per chip

- 60-135K patterns possible for the next generation chip (65-90 nm, custom cell, larger size, 3D technology)

1) Associative Memory design for the FastTrack processor (FTK) at ATLAS (ATL-DAQ-PROC-2010-013)

- Tree Search Processor (TSP) improves the resolution by a factor 2 to reject fake roads before track fitting

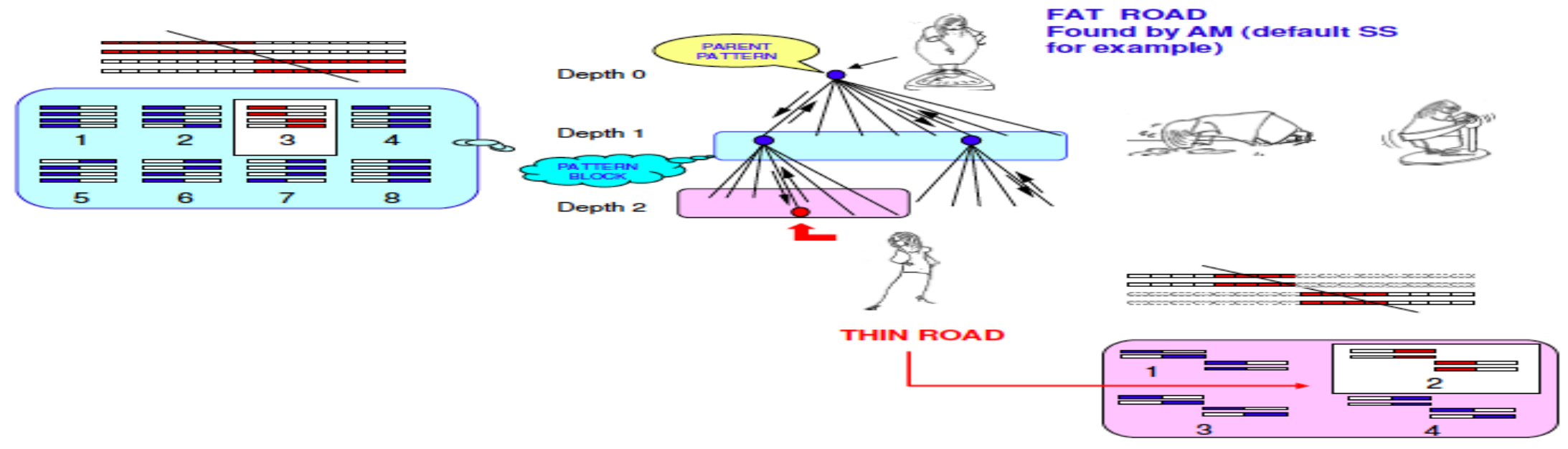




\section{Process Unit (AUX card)}

- Data Organizer (DO)

- Store full resolution hits in a smart database

- Send hits at a coarser resolution to the Associative Memory (AM) for pattern recognition

- Fetch the hits in a road and send to the Track Fitter for a matched pattern

- Track Fitter (TF)

- Use DSPs in an FPGA to calculate the helix parameters and the components of the $\chi^{2}$

- store the constants in the internal FPGA memory

- Expect ns per track with 288 DSPs in the FPGA being considered

- Hit Warrior (HW)

- Remove duplicate tracks defined as having more than $\mathbf{N}$ hits in common with another track

- Employ an associative memory built on the fly and implemented in an FPGA 


\section{Processor Unit Prototype}
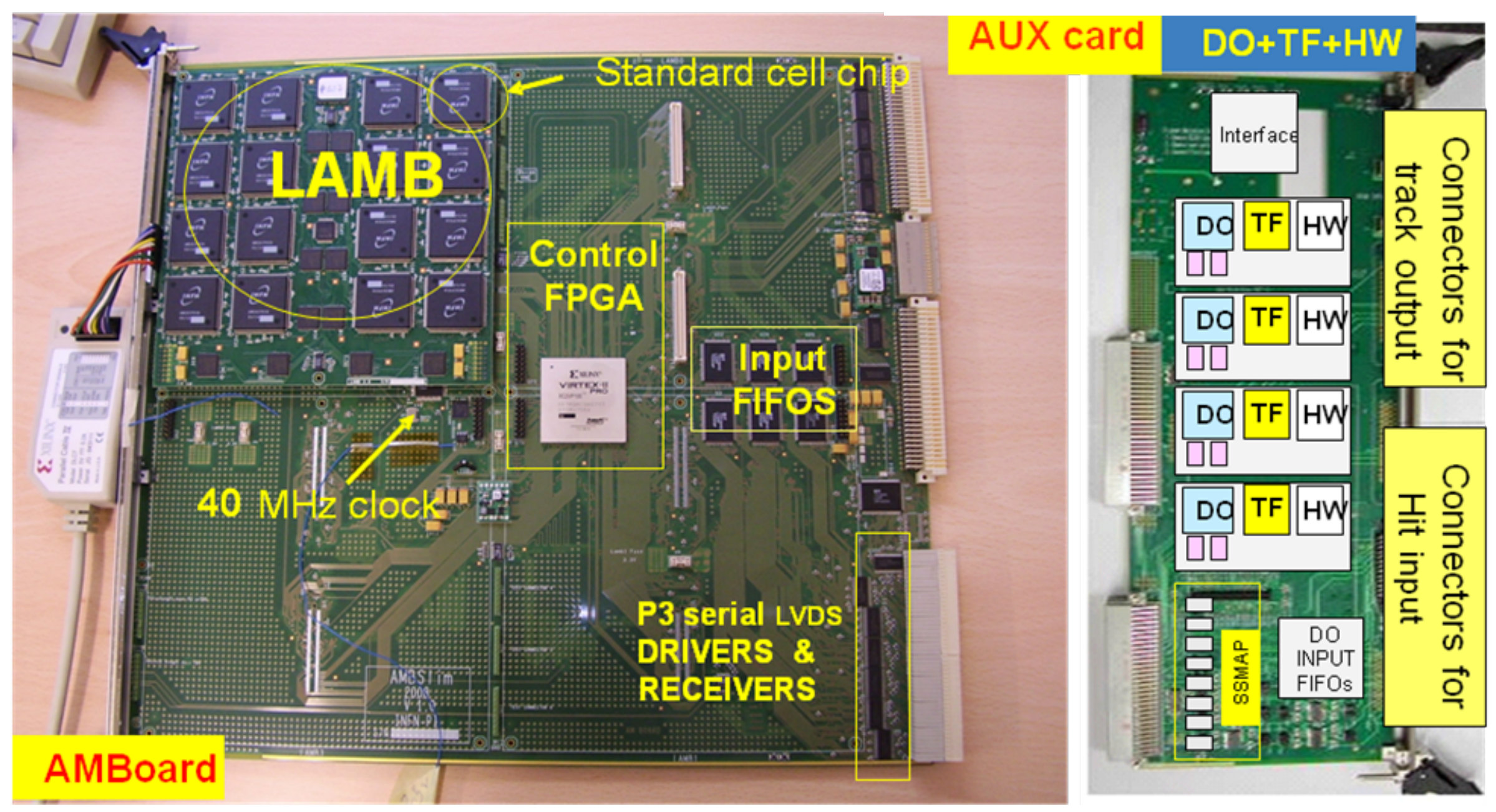


\section{Interface to the Current TDAQ}

- Input

- Dual output SLINK interface in the RODs of PIX and SCT

- Output

- ROD: The tracks from the second stage that pass a $\chi^{2}$ cut are sent to a ROD

- ROS: Special configuration or special running mode is necessary.

- The typical output event size for all tracks with PT $>1 \mathrm{GeV} / \mathrm{c}$ at $3 \times 10^{34}$ is $5.0 \mathrm{kB}$.

- Data is desirable at the full LVL1 rate for the LVL2 algorithms. 


\section{Single Track Efficiency}

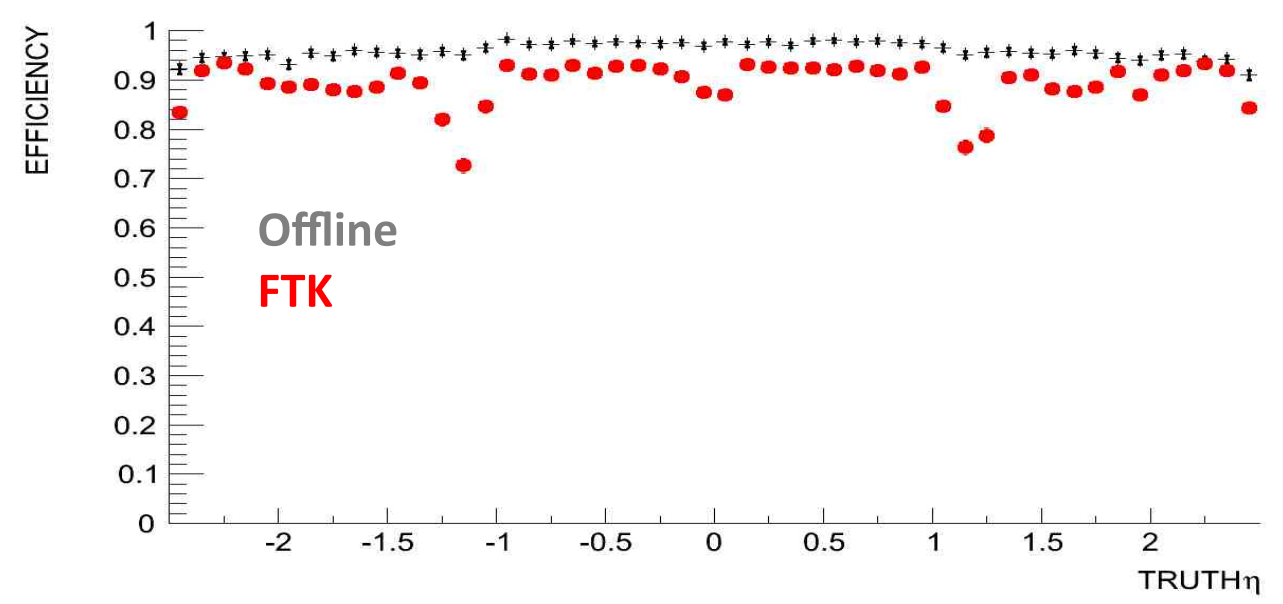

- The overall efficiency has not yet been optimized

- The dip at $\eta=0$ is an artifact of how we produced the pattern bank

- The dip near $|\eta|=1.2$, the region between the barrel and the forward, will be optimized 


\section{Timing}
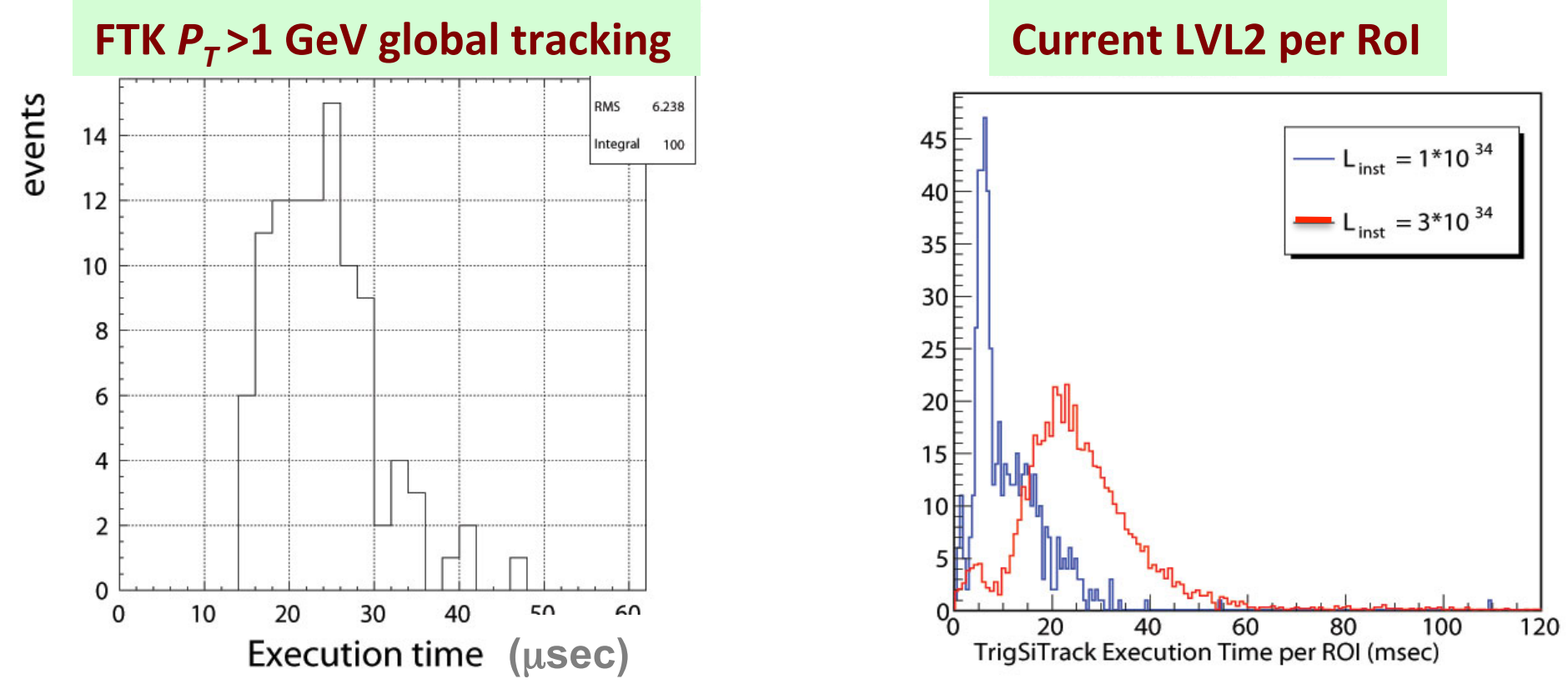

- WH(bb) MC events are used.

- FTK finishes global tracking in $25 \mu$ s at $3 \times 10^{34}$.

- Current LVL2 need 25 ms per jet or lepton Rol at $3 \times 10^{34}$ and the number of Rols is large 


\section{Summary}

- Global tracking can make a significant contribution to the ATLAS trigger.

- A conceptual design shows that an affordable FTK will take less than $100 \mu \mathrm{s} /$ event at the LHC design luminosity and beyond, and have excellent physics performance.

- The implementation can help ATLAS even at low luminosity. 


\section{Single Track Helix Parameter Resolution}
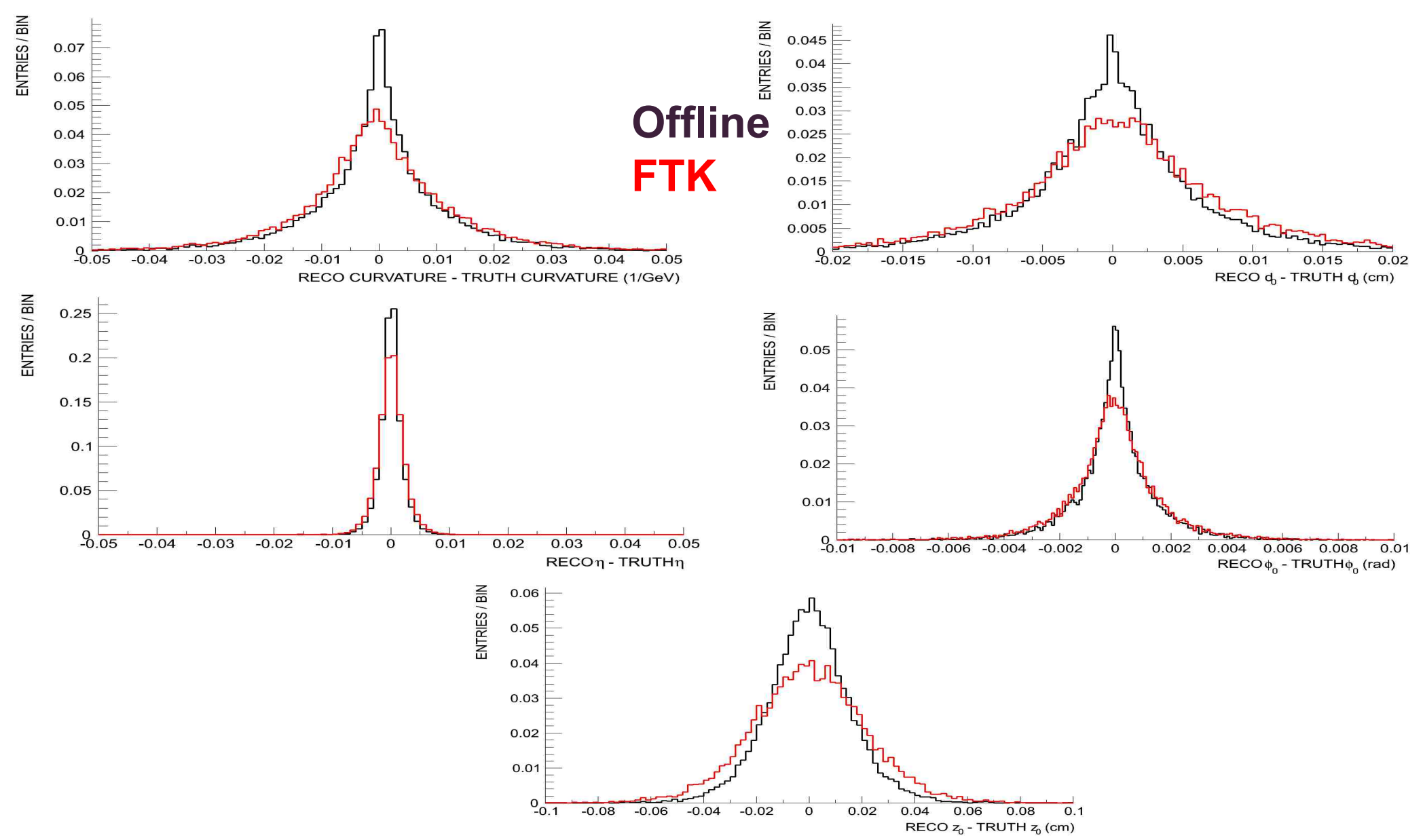


\section{$b$-tagging at $3 \times 10^{34}$}

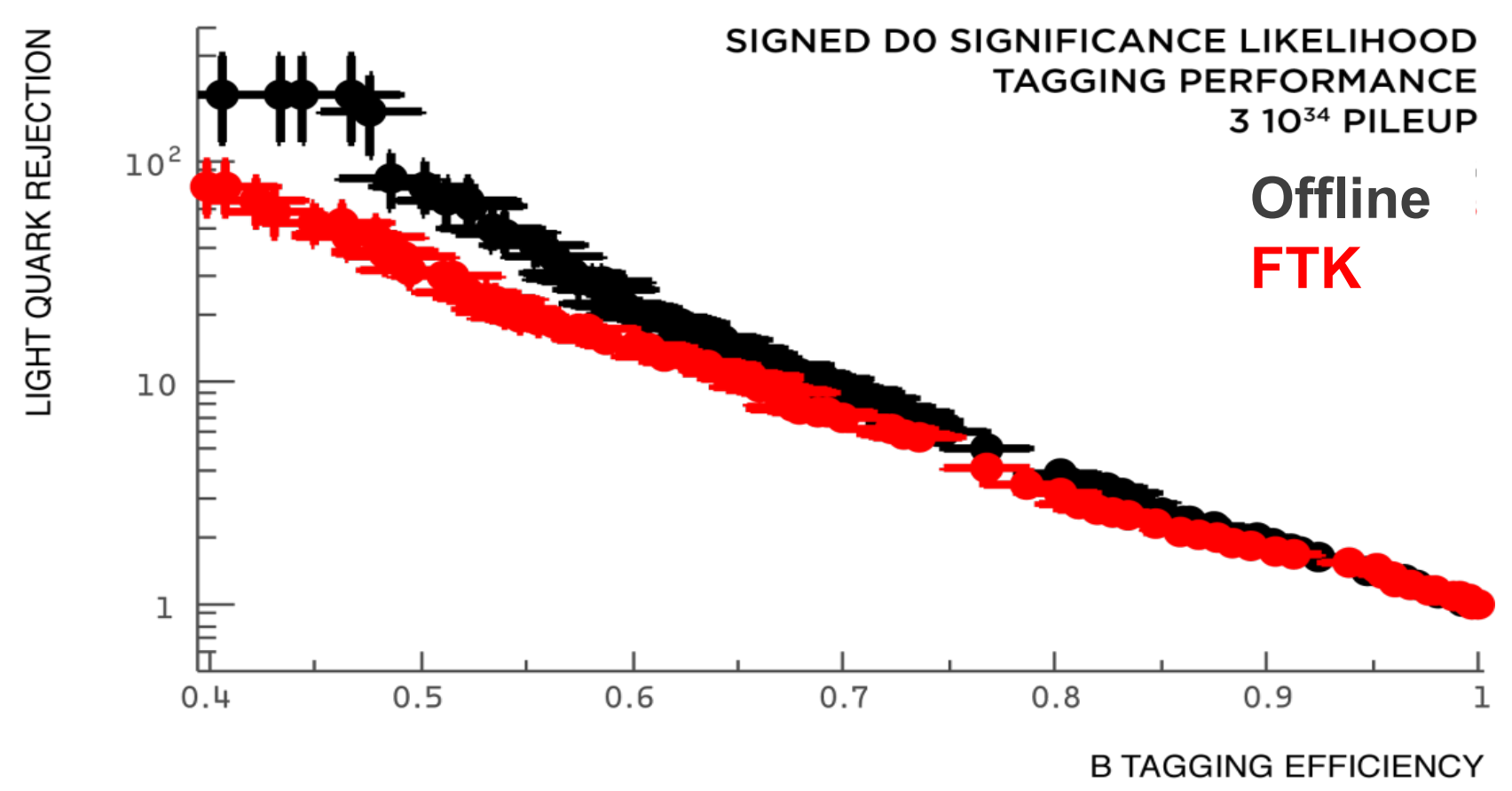

- Using signed impact-parameter significance likelihood tagger

- To test more sophisticated b-taggers to have a larger light-quark rejection 


\section{$\tau$-tagging at $3 \times 10^{34}$}
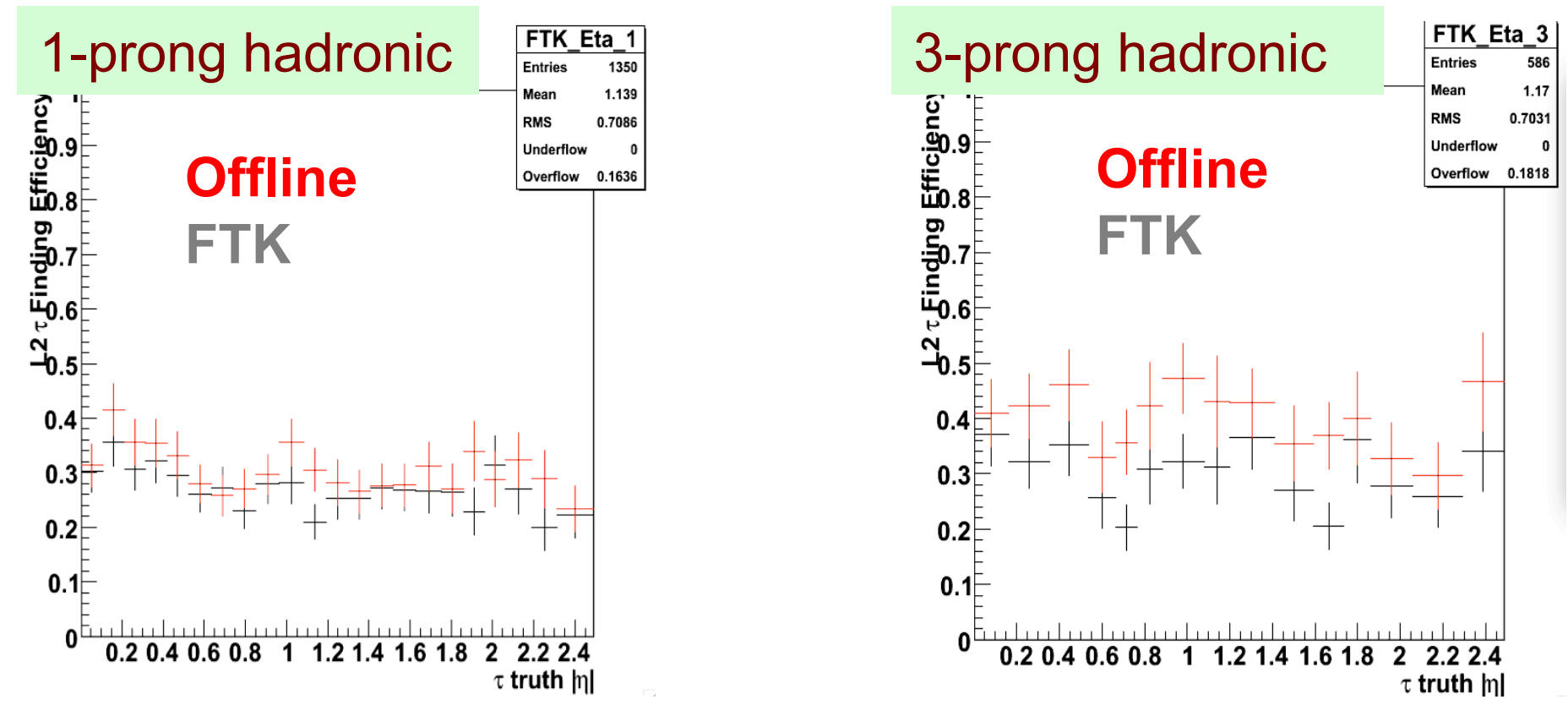

- Requiring 1 (2 or 3) tracks in the signal cone for 1 (3) prong $\tau$ 's and no tracks with $P_{T}>1.5 \mathrm{GeV} / \mathrm{c}$ in the isolation cone

- With $\sim 10^{-3}$ jet fake probability 


\section{Lepton (muon) Isolation at $3 \times 10^{34}$}
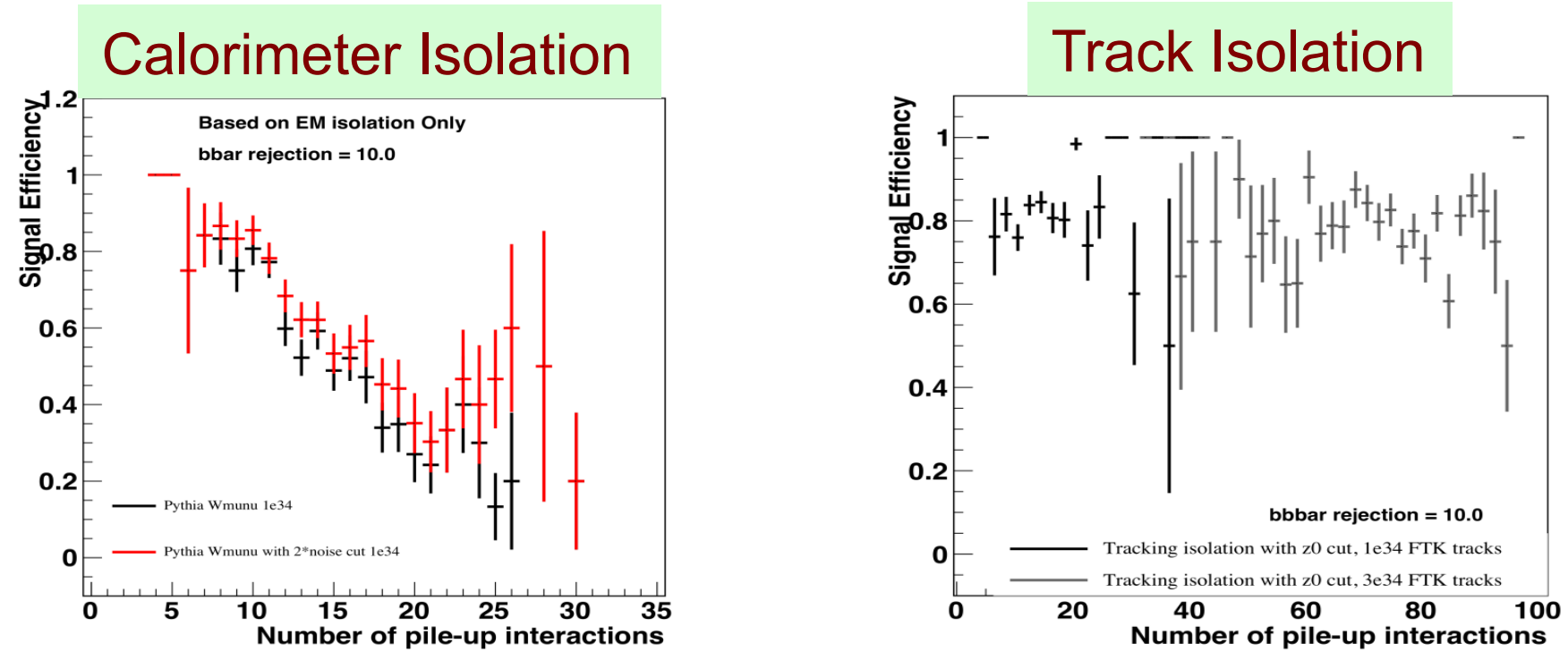

- Calorimeter isolation usually used to suppress QCD background but will deteriorate due to energy from 75 pileups at $3 \times 10^{34}$

- Track isolation will still work due to using only tracks pointing within a few $\mathrm{mm}$ of the muon at the beamline 